\title{
Actividad física y salud percibida en pacientes con enfermedad coronaria
}

\author{
Physical activity and perceived health in patients with coronary artery disease
}

\section{Actividade física e saúde percebida em pacientes com doença coronaria}

\author{
A. C. Stein ${ }^{1}$, O. Molinero ${ }^{1,3}$, A. Salguero', M. C. R. Corrêa $a^{1,2}$, S. Márquez ${ }^{1}$ \\ 1 Instituto de Biomedicina (IBIOMED) y Departamento de Educación Física, Universidad de León, España. 2 Facultad de Educación Física, Universidad \\ Federal de Goiás, Brasil. 3 Universidad Autónoma de Chile, Chile
}

Resumen: La enfermedad coronaria es una de las principales causas de morbilidad y mortalidad en el mundo y se asocia con importantes consecuencias emocionales y sociales. La actividad física parece ralentizar el inicio y la progresión de las enfermedades cardiovasculares pero también presenta beneficios sobre la salud psicosocial. El presente estudio tiene como objetivo el análisis de la relación entre la práctica de actividad física y la calidad de vida, el bienestar psicológico y la depresión en pacientes con enfermedad coronaria. La muestra estaba compuesta por 275 personas, con edades entre 54 a 98 años. Se aplicó un Cuestionario Sociodemográfico, el Cuestionario de Salud SF-36, la Escala de Depresión Geriátrica (GDS), la Escala de Bienestar Psicológico (EBP) y el Cuestionario de Actividad Física de Yale (YPAS). Los resultados obtenidos pusieron de manifiesto que la práctica de actividad física se asociaba en pacientes con enfermedad coronaria a una mejora en las distintas escalas del Cuestionario de Salud SF-36 y en la Escala de Depresión Geriátrica, confirmando su importancia para el mantenimiento de la calidad de vida relacionada con la salud en estos pacientes. Palabras clave: actividad física, enfermedad coronaria, depresión, bienestar psicológico.

Abstract: Coronary artery disease is one of the main causes of morbidity and mortality in the world and associates with important emotional and social consequences. Physical activity seems to slow down the start and the progression of the cardiovascular illnesses but also presents benfits on psychosocial health. The present study is amied to analyze the relationship between the practice of physical activity and quality of life, psychological wellbeing and depression in patients with coronary illness. The sample was composed by 275 subjects, aged between 54 to 98 years. They were applied a Sociodemograpahic Questionnaire, the Questionnaire of Health SF-36, the Scale of Geriatric Depression (GDS), the Scale of Psychological Wellbeing (EBP) and the Yale Physical Activity Scale (YPAS). Results obtained indicated that the practice of physical activity associated in patients with coronary illness to an improvement in the distinct scales of the Questionnaire of Health SF-36 and in the Scale of Geriatric Depression, confirming his importance for the maintenance of health-related quality of life in these patients.

Keywords: physical activity, coronary artery disease, depression, psychological wellbeing

Resumo: A doença coronaria é uma das principais causas de morbilidad e mortalidade no mundo e associa-se com importantes consequências emocionais e sociais. A actividade física parece reduzir o início e a progressão das doenças cardiovasculares mas também apresenta benefícios sobre a saúde psicosocial. O presente estudo tem como objectivo a análise da relaçấo entre a prática de actividade física e a qualidade de vida, o bem-estar psicológico e a depressão numa mostra de pacientes com doença coronaria. A mostra estava composta por 275 pessoas, com idades entre 54 a 98 anos. Aplicou-se um Cuestionario Sociodemográfico, o Cuestionario de Saúde SF-36, a Escala de Depressão Geriátrica (GDS), a Escala de Bem-estar Psicológico (EBP) e o Cuestionario de Actividade Física de Yale (YPAS). Os resultados obtidos puseram de manifesto que a prática de actividade física se associava em pacientes com doença coronaria a uma melhora nas diferentes escalas do Cuestionario de Saúde SF-36 e na Escala de Depressáo Geriátrica, confirmando sua importância para a manutenção da qualidade de vida relacionada com a saúde nestes pacientes.

Palabras chave: actividade física, doença coronaria, depressão, bem-estar psicológico.

\section{Introducción}

La enfermedad coronaria es una de las principales causas de morbilidad y mortalidad en el mundo y se asocia con importantes consecuencias emocionales y sociales (Cepeda, Cheong, Lee y Yan, 2011). Es un proceso de carácter multifactorial, con varios factores de riesgo establecidos, tales como índices elevados de colesterol, hipertensión y tabaquismo. Además de dichos factores, se ha observado que el estrés psicológico y la falta de actividad física pueden actuar como

Dirección para correspondencia [Correspodence address]: Olga Molinero González. Facultad de Ciencias de la Actividad Física y del Deporte, Universidad de León, Campus de Vegazana s/n, 24007 León. E-mail: olga.molinero@unileon.es desencadenantes para síndromes coronarios agudos (Thrall, Lane, Carroll y Lip, 2007). La actividad física es una conducta de salud importante para el tratamiento y prevención de la cardiopatía coronaria a todas las edades (Balady y cols., 2007; Boini, Briançon, Guillemin, Galan y Hercberg, 2006; Nguyen y cols., 2011; Shiroma y Lee, 2010). El equilibrio entre los riesgos y los beneficios de la práctica de actividad física en enfermos coronarios es claramente favorable a los segundos, sobre todo cuando la práctica es regular. Así, el ejercicio debe ser considerado como la piedra angular en la que han de basarse las modificaciones del estilo de vida para la prevención de la enfermedad cardiovascular (Pérez, 2008). Es un hecho bien constatado que la actividad física regular 
contribuye a la prevención primaria y secundaria de varias enfermedades crónicas y se asocia con un riesgo reducido de muerte prematura (Warburton y cols., 2006). La práctica de actividad física ha demostrado ser una estrategia eficaz para ralentizar el inicio y progresión de estas patología a través de efectos sobre aspectos tales como la adiposidad, la sensibilidad a la insulina, el control glucémico y la presión arterial (Bassuk y Manson, 2010). Pero, además, el ejercicio potencia los recursos motores en beneficio de la salud psicosocial, y de la autonomía e independencia en el desempeño de las actividades de la vida diaria (Sánchez, Ureña y Garcés de Los Fayos, 2002). Para García-Molina, Baeza y Fernández (2010), los beneficios psicosociales del ejercicio adquieren especial protagonismo, combatiendo el aislamiento, la depresión y la ansiedad y favoreciendo la autoestima y cohesión social. Por tal razón, diversos autores respaldan que la práctica de ejercicio físico regular a través de la inclusión en programas dirigidos o supervisados por especialistas del ámbito de la actividad física y respaldados por instituciones públicas, se presenta como la mejor terapia bio-psico-social (García-Molina y cols., 2010). Dichas prácticas físicas serían especialmente importantes en adultos mayores, favoreciendo un mejor estado de salud y calidad de vida que hiciesen factible mantener y/o favorecer la independencia funcional.

La salud relacionada con la calidad de vida se asocia, cada vez más, con un indicador de una buena de salud en la enfermedad coronaria (Cepeda y cols., 2011). Además de tener implicaciones importantes en otras enfermedades crónicas tales como diabetes, osteoporosis, obesidad y cáncer (Warburton, Nicol y Bredin, 2006), el bienestar psicológico es especialmente importante para la prevención y el tratamiento de las enfermedades cardiovasculares. Por otra parte, la depresión se asocia con frecuencia con las enfermedades cardiovasculares (Romero, 2007), y existe una estrecha relación entre la depresión y la calidad de vida en pacientes coronarios que han sufrido infarto de miocardio (Sandín, 2002).

En función de todos estos antecedentes, el presente estudio tiene como objetivo el análisis de la relación entre la práctica de actividad física y la calidad de vida, el bienestar psicológico y la depresión en una muestra de pacientes con enfermedad coronaria.

\section{Método}

\section{Participantes}

La muestra estaba compuesta por 275 sujetos con edades entre 60 a 98 años, con una media de edad de $74,9( \pm 8,2)$ ańos. En cuanto al género, incluía 95 hombres, con una media de edad de 76,1 $( \pm 7,6)$ ańos, y 180 mujeres con una media de edad de 74,4 $( \pm 8,5)$ años. Los sujetos se estratificaron según la presencia o ausencia de enfermedad coronaria partir de la in- formación obtenida mediante la aplicación de un cuestionario socio-demográfico. Asimismo, se clasificaron en Más Activos y Menos Activos en función del Índice de Actividad Total del cuestionario YPAS (Salguero, Martínez-García, Molinero y Márquez, 2011). En la tabla 1 se muestran el número de sujetos de cada grupo y su distribución según el género.

\section{Procedimiento}

La selección de los sujetos se llevó a cabo mediante el contacto con asociaciones que trabajan con personas mayores en la ciudad de León (España), así como con diversas residencias públicas y privadas para personas de edad avanzada. Se informó a los responsables, psicólogos y médicos sobre los objetivos del estudio, las características de los sujetos objeto de investigación, los diferentes cuestionarios psicológicos que iban a ser administrados y el tiempo necesario para llevar a cabo todo el proceso. A tal fin se les entregó un dossier con todas las informaciones necesarias. El estudio fue aprobado por el Comité Ético de la Universidad de León, y llevado a cabo con la conformidad de los respectivos responsables de las instituciones. Todos los participantes dieron su consentimiento por escrito para la participación en el estudio una vez que se les informó de los objetivos de la investigación y el protocolo a seguir.

\section{Instrumentos}

Se aplicó a los participantes un Cuestionario Sociodemográfico elaborado ad hoc, que además de otros datos generales permitió conocer diversos aspectos relacionados con la salud de los sujetos, concretamente la presencia o no de enfermedades coronarias (angina de pecho, infarto de miocardio, arritmia, hipertensión y tromboflebitis), así como sus hábitos de práctica de actividad física, como la participación de programas de actividad física regular.

Para analizar la percepción de la salud de los sujetos se utilizó el Cuestionario de Salud SF-36 (Ware y Sherbourne, 1992), en la versión española de Alonso, Prieto y Antó (1995). Hoy en día es el instrumento más empleado a nivel internacional para medir la calidad de vida relacionada con la salud (Ware, 2000). A través de los 36 ítems del cuestionario se valoran ocho dimensiones o escalas de la calidad de vida relacionada con la salud: Función Física, Rol Físico, Dolor Corporal, Función Social, Rol Emocional, Salud Mental, Vitalidad y Salud General.

La Escala de Depresión Geriátrica (GDS), específicamente diseñada para identificar la depresión en personas mayores, consta de 30 items y fue elaborada por Brink y cols. (1982) y Yesavage y cols. (1983). La versión española fue adaptada y validada por Izal y Montorio (1993). 
Tabla 1. Distribución de los sujetos en función de la presencia de enfermedad coronaria y el nivel de actividad física.

\begin{tabular}{lllll}
\hline Grupos & & Total & Hombres & Mujeres \\
\hline \multirow{2}{*}{ Más Activos } & Enfermedad Coronaria & 66 & 21 & 45 \\
\cline { 2 - 5 } & Sin Enfermedad Coronaria & 125 & 40 & 85 \\
\hline \multirow{2}{*}{ Menos Activos } & Enfermedad Coronaria & 40 & 15 & 25 \\
\cline { 2 - 5 } & Sin Enfermedad Coronaria & 44 & 19 & 25 \\
\hline
\end{tabular}

La Escala de Bienestar Psicológico (EBP), fue diseñada con el objetivo de evaluar el grado de felicidad o bienestar psicológico (Sánchez-Cánovas, 1998). Consta de 65 items que valora cuatro dimensiones, de las cuales solo utilizamos en el presente estudio el Bienestar Psicológico y el Bienestar Material.

Por último, el Cuestionario de Actividad Física de Yale (YPAS, Dipietro Caspersen, Ostfeld y Nadel, 1993), validada a la población española por De Abajo, Larriba y Márquez (2001), tiene el objetivo de evaluar la actividad física en personas mayores. Es un cuestionario de recuento de actividad física, que se lleva a cabo a través de una entrevista, en la que los sujetos especifican el tiempo utilizado en la realización de una serie de actividades físicas que se les presenta obteniéndose un total de 8 índices: Indice de Vigor (IV), Indice de Paseo
(IP), Indice de Movimiento General (IM), Indice de Estar de Pie (IE), Indice de Estar Sentado (IS), Indice de Tiempo Total Semanal (ITT), Indice del Gasto Energético Total Semanal (IGT), e Indice de Actividad Total Semanal (IAT).

\section{Análisis de datos}

El análisis de los datos se llevó a cabo mediante el paquete estadístico SSPS versión 19.0. Se realizó un análisis multivariante de la varianza (MANOVA) 2x2 (Nivel de actividad física x Enfermedad Coronaria) con todos los índices de salud percibida, depresión, bienestar psicológico y actividad física. Para estudiar las diferencias específicas entre distintos grupos, se llevó a cabo un análisis post-hoc mediante el procedimiento de Bonferroni.

Tabla 2. Análisis MANOVA en relación a la presencia de enfermedad coronaria y el nivel de actividad física

\begin{tabular}{|c|c|c|c|c|c|c|c|c|}
\hline & \multirow[b]{2}{*}{ Escalas } & \multicolumn{2}{|c|}{ Enfermedad Coronaria } & \multicolumn{2}{|c|}{ Actividad Física } & \multirow[b]{2}{*}{$\begin{array}{l}\text { Enfermedad } \\
\text { Coronaria }\end{array}$} & \multicolumn{2}{|l|}{ Efectos } \\
\hline & & $F$ & $p$ & $F$ & $p$ & & $\begin{array}{l}\text { Actividad } \\
\text { Fisica }\end{array}$ & $\begin{array}{l}\text { Enfermedad Coronaria } \\
x \text { Actividad Física }\end{array}$ \\
\hline \multirow{8}{*}{ SF-36 } & Función Física & 7,56 & 0,006 & 21,40 & 0,001 & 0,027 & 0,080 & 0,106 \\
\hline & Rol Físico & 17,94 & 0,000 & 4,01 & 0,046 & 0,062 & 0,013 & 0,070 \\
\hline & Dolor Corporal & 9,71 & 0,002 & 0,86 & 0,355 & 0,033 & 0,002 & 0,037 \\
\hline & Salud General & 10,74 & 0,001 & 5,15 & 0,024 & 0,038 & 0,004 & 0,074 \\
\hline & Vitalidad & 3,90 & 0,049 & 1,66 & 0,198 & 0,014 & 0,007 & 0,031 \\
\hline & Función Social & 0,84 & 0,361 & 0,25 & 0,618 & 0,003 & 0,001 & 0,029 \\
\hline & Rol Emocional & 3,28 & 0,071 & 0,24 & 0,625 & 0,012 & 0,001 & 0,018 \\
\hline & Salud Mental & 8,66 & 0,004 & 9,56 & 0,002 & 0,031 & 0,024 & 0,075 \\
\hline \multirow{2}{*}{ GDS } & Puntuación Depresión & 6,61 & 0,011 & 9,77 & 0,002 & 0,024 & 0,019 & 0,083 \\
\hline & Nivel Depresión & 5,42 & 0,021 & 8,31 & 0,004 & 0,019 & 0,018 & 0,057 \\
\hline \multirow{2}{*}{ EBP } & Bienestar Subjetivo & 6,35 & 0,012 & 2,39 & 0,123 & 0,023 & 0,018 & 0,066 \\
\hline & Bienestar Material & 0,017 & 0,898 & 3,02 & 0,083 & 0,001 & 0,009 & 0,074 \\
\hline \multirow{8}{*}{ YPAS } & ITT (h/sem) & 11,26 & 0,001 & 20,77 & 0,001 & 0,040 & 0,126 & 0,100 \\
\hline & IGT (kcal/sem) & 10,12 & 0,002 & 34,84 & 0,001 & 0,036 & 0,153 & 0,135 \\
\hline & IV & 3,61 & 0,059 & 41,29 & 0,001 & 0,013 & 0,210 & 0,140 \\
\hline & IP & 2,75 & 0,099 & 6,07 & 0,014 & 0,010 & 0,034 & 0,030 \\
\hline & IM & 9,07 & 0,003 & 12,51 & 0,001 & 0,032 & 0,064 & 0,071 \\
\hline & IE & 7,64 & 0,006 & 62,17 & 0,001 & 0,027 & 0,258 & 0,204 \\
\hline & IS & 3,23 & 0,073 & 6,62 & 0,110 & 0,012 & 0,046 & 0,035 \\
\hline & IAT & 1,14 & 0,286 & 18,87 & 0,001 & 0,004 & 0,101 & 0,071 \\
\hline
\end{tabular}

IV = Índice de Vigor; IP = Índice de Paseo; IM = Índice de Movimiento General; IE = Índice de Estar de Pie; IS = Índice de Estar Sentado); ITT = Índice de Tiempo Total Semanal; IGT = Índice de Gasto Energético Total Semanal; IAT = Índice de Actividad Total Semanal. 


\section{Resultados}

Se detectaron efectos significativos según la presencia o ausencia de enfermedad coronaria para el Cuestionario $S F$ 36 ( $\lambda$ Wilks=0,908, F(8)=3,36, p=0,001), la Escala GDS $(\lambda$ Wilks=0,976, $\mathrm{F}(2)=3,29, \mathrm{p}=0,039)$, la Escala EBP $(\lambda \mathrm{Wi}-$ $\mathrm{lks}=0,960, \mathrm{~F}(2)=5,63, \mathrm{p}=0,001)$ y el Cuestionario $Y P A S(\lambda$ Wilks=,928, $\mathrm{F}(8)=2,570, \mathrm{p}=0,01)$. En relación a la práctica de actividad física se encontraron los siguientes efectos: Cuestionario $S F-36(\lambda$ Wilks=0,860, $\mathrm{F}(8)=5,41, \mathrm{p}=0,001)$, Escala GDS $(\lambda$ Wilks=0,965, $\mathrm{F}(2)=4,88, \mathrm{p}=0,008)$, Escala EBP $(\lambda$ Wilks=0,988, $\mathrm{F}(2)=1,62, \mathrm{p}=0,198)$ y Cuestionario $Y P A S(\lambda$ Wilks=0,726, $\mathrm{F}(8)=12,54, \mathrm{p}=0,001)$. La Tabla 2 muestra los resultados del análisis MANOVA para cada una de las escalas de los distintos cuestionarios.

De acuerdo con los resultados presentados en la tabla 3, observamos que el grupo Menos Activo con Enfermedad Co- ronaria presentó una peor puntuación de la percepción de la salud en todos los factores del SF-36 en comparación con los sujetos Menos Activos sin Enfermedad Coronaria. También alcanzaron puntuaciones más bajas respecto a los sujetos Más Activos con Enfermedad Coronaria en los factores Función Física, Salud General, Vitalidad y Salud Mental. En comparación con el grupo Más Activo sin Enfermedad Coronaria puntuaron más bajo en Función Física, Salud General, Vitalidad y Salud General.

En lo que hace referencia a la Escala de Depresión Gerátrica, se puso de manifiesto que las personas del grupo Menos Activos con Enfermedad Coronaria eran las que presentaban mayores índices de depresión en comparación con los grupos Más Activos con Enfermedad Coronaria, Más Activos Sin Enfermedad Coronaria y Menos Activos sin Enfermedad Coronaria.

Tabla 3. Puntuaciones medias de los Cuestionarios SF-36, GDS, EBP y YPAS para los distintos grupos.

\begin{tabular}{|c|c|c|c|c|c|c|}
\hline & & Menos Activos & & Más Activos & & $p$ \\
\hline & Escalas & Enfermedad Coronaria & Sin Enfermedad Coronaria & Enfermedad Coronaria & Sin Enfermedad Coronaria & \\
\hline \multirow{8}{*}{ SF-36 } & Función Física & $59,88 \pm 23,73$ & $74,32 \pm 25,44$ & $79,17 \pm 21,78$ & $81,20 \pm 18,33$ & $\mathrm{a}, \mathrm{b}, \mathrm{c}$ \\
\hline & Rol Físico & $73,75 \pm 40,81$ & $89,20 \pm 27,70$ & $80,30 \pm 37,60$ & $94,80 \pm 19,39$ & $\mathrm{a}, \mathrm{c}, \mathrm{d}$ \\
\hline & Dolor Corporal & $61,00 \pm 28,27$ & $72,50 \pm 25,62$ & $65,45 \pm 23,35$ & $72,00 \pm 17,32$ & $\mathrm{~b}, \mathrm{~d}$ \\
\hline & Salud General & $45,63 \pm 23,15$ & $61,59 \pm 20,93$ & $57,80 \pm 16,32$ & $60,80 \pm 17,43$ & $a, b, c$ \\
\hline & Vitalidad & $59,38 \pm 20,13$ & $71,25 \pm 22,44$ & $68,26 \pm 19,99$ & $69,68 \pm 21,48$ & $\mathrm{~b}, \mathrm{~d}$ \\
\hline & Función Social & $83,13 \pm 21,66$ & $93,19 \pm 15,12$ & $90,91 \pm 16,63$ & $88,80 \pm 16,56$ & a \\
\hline & Rol Emocional & $81,67 \pm 31,08$ & $94,70 \pm 17,53$ & $83,84 \pm 34,71$ & $88,00 \pm 30,35$ & \\
\hline & Salud Mental & $59,80 \pm 22,97$ & $74,72 \pm 19,91$ & $74,24 \pm 22,28$ & $76,90 \pm 18,32$ & $a, b, c$ \\
\hline \multirow{2}{*}{ GDS } & Puntuac. Depresión & $12,30 \pm 7,16$ & $7,23 \pm 6,38$ & $7,15 \pm 6,22$ & $7,03 \pm 5,91$ & $\mathrm{a}, \mathrm{b}, \mathrm{c}$ \\
\hline & Nivel Depresión & $1,68 \pm 0,73$ & $1,34 \pm 0,53$ & $1,32 \pm 0,56$ & $1,27 \pm 0,50$ & $a, b, c$ \\
\hline \multirow{2}{*}{ EBP } & Bienest. subjetivo & $103,95 \pm 21,61$ & $120,98 \pm 16,72$ & $107,73 \pm 18,33$ & $109,45 \pm 19,90$ & $\bar{a}$ \\
\hline & Bienest. material & $37,58 \pm 8,68$ & $44,35 \pm 5,00$ & $40,95 \pm 6,97$ & $38,22 \pm 9,68$ & $\mathrm{a}$ \\
\hline \multirow{8}{*}{ YPAS } & ITT (h/sem) & $21,89 \pm 11,29$ & $31,08 \pm 23,04$ & $34,85 \pm 19,04$ & $41,57 \pm 23,48$ & $\mathrm{~b}, \mathrm{c}, \mathrm{d}$ \\
\hline & IGT (kcal/sem) & $4180 \pm 2505$ & $5524 \pm 3802$ & $7323 \pm 4090$ & $8804 \pm 5095$ & $\mathrm{~b}, \mathrm{c}, \mathrm{d}$ \\
\hline & IV & $9,10 \pm 18,01$ & $18,86 \pm 24,96$ & $36,21 \pm 30,86$ & $37,76 \pm 28,67$ & $\mathrm{~b}, \mathrm{c}$ \\
\hline & IP & $33,50 \pm 13,85$ & $32,18 \pm 13,31$ & $29,64 \pm 19,94$ & $25,80 \pm 18,58$ & d \\
\hline & IM & $9,65 \pm 2,66$ & $11,25 \pm 2,60$ & $11,45 \pm 3,28$ & $12,20 \pm 3,30$ & $a, b, c$ \\
\hline & IE & $4,45 \pm 1,78$ & $5,64 \pm 2,13$ & $7,24 \pm 2,37$ & $7,54 \pm 2,41$ & $\mathrm{~b}, \mathrm{c}$ \\
\hline & IS & $3,10 \pm 0,87$ & $2,75 \pm 0,78$ & $2,65 \pm 0,94$ & $2,54 \pm 1,12$ & \\
\hline & IAT & $59,88 \pm 26,86$ & $70,68 \pm 30,42$ & $87,20 \pm 43,89$ & $85,82 \pm 36,81$ & $\mathrm{~b}, \mathrm{c}$ \\
\hline
\end{tabular}

IV = Índice de Vigor; IP = Índice de Paseo; IM = Índice de Movimiento General; IE = Índice de Estar de Pie; IS = Índice de Estar Sentado); ITT = Índice de Tiempo Total Semanal; IGT = Índice de Gasto Energético Total Semanal; IAT = Índice de Actividad Total Semanal.

Los datos son medias y desviación típica. P $<0,05$. a = Menos Activos Con Enfermedad Coronaria/Menos Activos Sin Enfermedad Coronaria; b = Menos Activos Con Enfermedad Coronaria/Más Activos Con Enfermedad Coronaria; c = Menos Activos Con Enfermedad Coronaria/Más Activos Sin Enfermedad Coronaria; d= Más Activos Con Enfermedad Coronaria/Más Activos Sin Enfermedad Coronaria. 
En cuanto a la Escala EBP existía una diferencia significativa en el Bienestar Psicológico a favor del grupo Menos Activo sin Enfermedad Coronaria en relación a los grupos Más Activos enfermos y sanos, y también en relación al grupo Menos Activo con patología $(\mathrm{p}=0,001)$. De igual manera, era este mismo grupo de personas quienes presentaba un mejor Bienestar Material, con diferencias significativa en relación a los grupos Más Activos sanos y Menos Activos que padecen enfermedad.

En el Cuestionario YPAS, también se observaron diferencias significativas. En la práctica totalidad de los índices, a excepción de IP e IS, los sujetos del grupo Menos Activo con Enfermedad Coronaria alcanzó puntuaciones más bajas que los sujetos de los grupos Más Activos sanos y enfermos y las puntuaciones también mostraron una tendencia a ser más bajas respecto al grupo Menos Activo sin Enfermedad Coronaria. Tanto en los índices ITT como GT los sujetos Más activos sanos puntuaron más alto que los enfermos.

\section{Discusión}

El análisis de los resultados del Cuestionario SF-36 puso de manifiesto que el grupo Menos Activo con Enfermedad Coronaria fue el que presentó puntuaciones más bajas en la práctica totalidad de factores, lo que nos indica que padecer esta enfermedad puede influir en la percepción de la salud, y mantener una vida sedentaria también puede traducirse en una mala percepción de la salud, potenciándose la combinación de estas dos condiciones. El estudio longitudinal realizado por Boini y cols. (2006), con el objetivo de investigar el impacto de la presencia de enfermedad coronaria en la salud relacionada con la calidad de vida, llevado a cabo en una población de adultos de 35 a 60 ańos, puso de manifiesto que, después de aproximadamente 2,5 años del diagnóstico de la enfermedad arterial coronaria, los aspectos con mayor probabilidad de ser afectados, fueron los factores Función Física, Rol Emocional, Rol Físico, Vitalidady Salud General. Nuestros resultados son similares en parte, aunque también se detectan efectos significativos en la escala de Salud Mental. Dichos efectos coinciden con los resultados de la escala GDS, que indican que los dos grupos de personas físicamente activas son las que menos sufren de depresión, lo que sugiere que el ejercicio tiene un efecto antidepresivo tanto para las personas que no presentan enfermedad coronaria como en pacientes (Salguero y cols., 2011). En la literatura encontramos estudios que han demostrado que los síntomas depresivos están asociados con un mayor riesgo de problemas cardíacos (Barth, Schumacher y Herrmann, 2004; Sorensen, Friis, Haghfelt y Bech, 2005), y que los pacientes con enfermedad coronaria sufren altas tasas (entre 15 y 30\%) de trastornos psicológicos (Ladwig y cols., 2008; Thombs y cols., 2005). Para Aguilar y Ávila (2007) la depresión geriátrica es una enfermedad debida, entre otros, al aumento de la esperanza de vida de la población y al desarrollo de enfermedades y de discapacidad, siendo el ejercicio físico, practicado de manera apropiada, la mejor herramienta disponible actualmente para retrasar y prevenir las consecuencias del envejecimiento, así como para fomentar la salud y el bienestar de las personas (Garzón, Porce y Ruiz, 2005). Los resultados hallados en el estudio de Wilson, Sánchez-Rodríguez y Mendoza (2009), que tenían el objetivo de determinar la relación entre el ejercicio físico con la depresión en ancianos, exponen una prevalencia significativamente más alta de depresión en el grupo de adultos mayores menos activos en comparación con los que realizan ejercicio físico. Según Murillo y Loo (2007) los adultos mayores que practican ejercicio mantienen una mayor funcionalidad física y mental en comparación con los menos activo. Nuestros resultados también coinciden con lo indicado por Stafford, Berk, Reddy y Jackson (2007), quienes concluyeron que en los pacientes con enfermedad arterial coronaria existe una prevalencia desproporcionadamente alta de depresión, con un efecto adverso en la calidad de vida, que a su vez se asocia con un aumento en la mortalidad.

Knechtle (2004) destaca que el ejercicio realizado regularmente es capaz de mejorar significativamente el bienestar psicológico de las personas de ambos géneros y a cualquier edad. Sin embargo, los resultados de nuestro estudio no llegan a poner de manifiesto un efecto positivo del ejercicio sobre el mismo. Constatamos que el grupo Menos Activo sin Enfermedad Coronaria es el que presenta un mejor bienestar psicológico y parece que la presencia de enfermedad coronaria es el principal del mismo, visto que los dos grupos que presentan enfermedad coronaria son los que tienen una peor percepción del bienestar psicológico. Estos resultados pueden justificarse debido al impacto del uso de medicamentos y sus efectos colaterales, así como el grado de limitaciones causadas por la enfermedad que, pueden afectar directamente las actividades de la vida diaria (Benetti, Nahas, Rebelo, Lemos y Carvalho, 2001). Además, nos encontramos ante una enfermedad crónica, cuyo tratamiento inadecuadao puede implicar en un agravamiento, causando una progresiva incapacidad física y consecuentemente empeorando de la calidad de vida (Giannotti, 2002).

Al profundizar en los aspectos relacionados con la práctica de actividad física a través de los resultados obtenidos en el Cuestionario YPAS, se ponía de manifiesto que el grupo Activo sin Enfermedad Coronaria, es el que dedica más horas semanales a la práctica de ejercicio, presentando un gasto calórico más elevado, una suma mayor de horas de tiempo activo, un mayor tiempo de permanencia en movimiento, y menos horas sentados, y realizan actividades más vigorosas. Riebe y cols. (2005) afirman que cuanto más tiempo se emplea a la realización de actividad física de forma regular, mayor es el gasto energético, lo que representa, mayores valores en el Índice de Gasto Energético Semanal. Nuestros resultados se 
encuentran en la misma línea de los obtenidos por Hillman, Kramer, Belopolsky y Smith (2006), quienes en una muestra dividida en un grupo de sujetos Menos Activo y otro de sujetos físicamente Activos, y mostraron que, los Índices de Tiempo Total Semanal, Gasto Energético Total Semanal y Actividad Física Total Semanal, alcanzaron puntuaciones significativamente más elevadas en las personas activas, frente a los Menos Activo. En la literatura, encontramos investigaciones que corroboran con estos resultados, comprobando que las personas mayores más independientes físicamente, mantienen un mayor nivel de actividad física, obteniendo puntuaciones más elevadas que las personas menos activas en prácticamente todos los índices del Cuestionario YPAS (Garatachea y cols., 2009; Harada, Chiu, King y Stewart, 2001; Salguero y cols., 2010; Wall, Ballard, Troped, Njike y Katz, 2010).

Según Durstine, Gordon, Wang y Luo (2012), las enfermedades crónicas son la principal causa de muerte en el mun- do, y las tasas de incidencia continúan aumentando siendo que, este aumento está fuertemente asociado con la inactividad física. Mantener un buen bienestar físico es importante para los pacientes en condiciones crónicas, tales como las enfermedades del corazón, porque la disminución de la capacidad física y el funcionamiento se asocian con un mayor uso de la atención primaria de salud, la morbilidad y mortalidad (Bayliss, Bayliss, Ware Jr y Steiner, 2004). La enfermedad coronaria puede afectar negativamente la vida de las personas mayores a nivel físico, psicológico y social, acarreando como consecuencia el empeoramiento de la calidad de vida de las personas que sufren esta patología. Los resultados obtenidos en este estudio confirman que la práctica habitual de actividad física puede ayudar a mejorar la percepción de la salud en presencia de enfermedad coronaria y actuar como un antidepresivo natural, siendo fundamental para mantener la calidad de vida relacionada con la salud.

\section{Referencias}

1. Aguilar, S. \& Ávila, J. A. (2007). La depresión: particularidades clínicas y consecuencias en el adulto mayor. Gaceta Médica de México, 143 (2), 141-148.

2. Alonso, J., Prieto, L. \& Antó, J. M. (1995). La versión española del SF36 health survey (cuestionario de salud SF-36): un instrumento para la medida de los resultados clínicos. Medicina Clínica, 104 (20), 771-776.

3. Balady, G. J., William, A. K., Ades, P. A., Bittner, V., Comoss, P., Foody, J. M., Franklin, B., Sanderson, B. \& Southard, D. (2007). American Heart Association/AACVPR scientific statement: core components of cardiac secondary prevention program. Circulation, 115, 2675-2682.

4. Barth, J., Schumacher, M. \& Herrmann, C. (2004). Depression as a risk factor for mortality in patients with coronary heart disease: a metaanalysis. Psychosomatic Medicine, 66, 802-813.

5. Bassuk, S. S. \& Manson, J. E. (2010). Physical activity and cardiovascular disease prevention in women: a review of the epidemiologic evidence. Nutrition, Metabolism \& Cardiovascular Diseases, 20, 467-473.

6. Bayliss, E. A., Bayliss, M. S., Ware Jr, J. E. \& Steiner, J. F. (2004). Predicting declines in physical function in persons with multiple chronic medical conditions: what we can learn from the medical problem list. Health and Quality of Life Outcomes, 2 (47), 1-8.

7. Benetti, M., Nahas, M. V., Rebelo, F. P. V., Lemos, L. S. \& Carvalho, T. (2001). Alteraçóes na qualidade de vida em coronariopatas acometidos de infarto agudo do miocárdio, submetidos a diferentes tipos de tratamentos. Atividade Física \& Saúde, 6 (3), 27-33.

8. Boini, S., Briançon, S., Guillemin, F., Galan, P. \& Hercberg, S. (2006). Occurrence of coronary artery disease has an adverse impact on healthrelated quality of life: a longitudinal controlled study. International Journal of Cardiology, 113, 215- 222.

9. Brink, T. L., Yasavage, J. A., Lum, O., Heersema, P. H., Adey, M. \& Rose, T. C. (1982). Screening tests for geriatric depression. Clinical Gerontologist, 1 (1), 37-43.

10. Cepeda, B., Cheong, A. P., Lee, A. \& Yan, B. P. (2011). Measuring health related quality of life in coronary heart disease: the importance of feeling well. International Journal of Cardiology, 149, 4-9.

11. De Abajo, S., Larriba, R. \& Márquez, S. (2001). Validity and reability of the yale physical activity survey in spanish elderly. Journal of Sport Medicine and Physical Fitness, 41 (4), 479-485.

12. Dipietro, L., Caspersen, C. J., Ostfeld, A. M. \& Nadel, E. R. (1993). A survey for assessing physical activity among older adults. Medicine \&
Science in Sports \& Exercise, 25, 628-642.

13. Durstine, J. L., Gordon, B., Wang, Z. \& Luo, X. (2012). Chronic disease and the link to physical activity. Journal of Sport and Health Science, 7, 1-9.

14. Garatachea, N., Molinero, O., Martínez-García, R., Jiménez-Jiménez, R., González-Gallego, J. \& Márquez, S. (2009). Feelings of well being in elderly people: relationship to physical activity and physical function. Archives of Gerontology and Geriatrics, 48 (3), 306-312.

15. García-Molina, A. V. A., Baeza, A. \& Fernández, M. (2010). Beneficios de la actividad física en personas mayores. Revista Internacional de $\mathrm{Me}$ dicina y Ciencias de la Actividad Física y del Deporte, 10 (40), 556-576.

16. García-Molina, A. V. A., Carbonell, A. \& Delgado, M. (2010). Beneficios de la actividad física en personas mayores. Revista Internacional de Medicina y Ciencias de la Actividad Física y del Deporte, 10 (40), 556-576.

17. Garzón, M. J. C., Porce, F. B. O. \& Ruiz, J. R. (2005). Mejora de la forma física como terapia antienvejecimiento. Medicina Clínica, 124 (4), 146-155.

18. Giannotti, A. (2002). Prevenção da doença coronária: perspectiva psicológica em um programa multiprofissional. Psicología USP, 13 (1), 1-22.

19. Harada, N. D., Chiu0, V., King, A. C. \& Stewart, A. L. (2001). An evaluation of three self-report physical activity instruments for older adults. Medicine \& Science in Sports \& Exercise, 33 (6), 962-970.

20. Hillman, C. H., Kramer, A. F., Belopolsky, A. V. \& Smith, D. P. (2006a). A cross-sectional examination of age and physical activity on performance and event-related brain potentials in a task switching paradigm. International Journal of Psychophysiology, 59 (4), 30-39.

21. Izal, M. \& Montorio, I. (1993). Adaptation of the Geriatric Depression Scale in Spain. Clinical Gerontologist, 13 (2), 83-91.

22. Knechtle, B. (2004). Der günstige einfluss von körperlicher aktivität auf wohlbefinden und psyche. Praxis, 93, 1403-1411.

23. Ladwig, K. H., Lederbogen, F., Völler, H., Albus, C., Hermann, C., Jordan, J., Köllner, V., Jünger, J., Lange, H., \& Fritzsche, K. (2008). Positionspapier zur bedeutung von psychosozialen faktoren in der kardiologie. Der Kardiologe, 2 (4), 274-287.

24. Murillo, A. \& Loo, I. (2007). Influencia de la práctica del ejercicio en la funcionalidad física y mental del adulto mayor. Revista de Enfermería del Instituto Mexicano del Seguro Social, 15 (1), 11-20.

25. Nguyen, P. K., Terashima, M., Fair, J. M., Varady, A., Taylor, R. E., Iribarren, C., Go, A. S., Haskell, W. L., Hlatky, M. A., Fortmann, S. 
P. \& McConnell, M. V. (2011). Physical activity in older subjects is associated with increased coronary vasodilation. JACC: Cardiovascular Imaging, 4 (6), 622-629.

26. Pérez, A. B. (2008). Ejercicio, piedra angular de la prevención cardiovascular. Revista Española de Cardiología, 61 (5), 514-528.

27. Riebe, D., Garber, C. E., Rossi, J. S., Greaney, M. L., Nigg, C. R., Lees, F. D., Burbank, P. M. \& Clark, P. G. (2005). Physical activity, physical function, and stages of change in older adults. American Journal of Health Behavior, 29 (1), 70-80.

28. Romero, C. E. (2007). Depresión y enfermedad cardiovascular. Revista Uruguaya de Cardiología, 22 (2), 1-16.

29. Salguero, A., Martínez-García, R., Molinero, O., Márquez, S. (2011). Physical activity, quality of life and symptoms of depression in community-dwelling and institutionalized older adults. Archives of Gerontology and Geriatrics 53, 152-157.

30. Sánchez, P. A., Ureña, F. \& Garcés de Los Fayos, E. J. (2002). Repercusiones de un programa de actividad física gerontológica sobre la aptitud física, autoestima, depresión y afectividad. Cuadernos de Psicología del Deporte, 2 (2), 57-73.

31. Sánchez-Cánovas, J. (1998). Escala de bienestar psicológico. Madrid, España: TEA Ediciones S.A.

32. Sandin, B. (2002). Papel de las emociones negativas en el trastorno cardiovascular: un análisis crítico. Revista de Psicopatología y Psicología Clinica, 7 (1), 1-18.

33. Shiroma, E. J. \& Lee, I. M. (2010). Physical activity and cardiovascular health: lessons learned from epidemiological studies across age, gender, and race/ethnicity. Circulation, 122, 743-752.

34. Sorensen, C., Friis, E., Haghfelt, T. \& Bech, P. (2005). Postmyocardial infarction mortality in relation to depression: a systematic critical review. Psychother Psychosom, 74, 69-80.

35. Stafford, L., Berk, M., Reddy, p. \& Jackson, H. J. (2007). Comorbid depression and health-related quality of life in patients with coronary artery disease. Journal of Psychosomatic Research, 62, 401-410.

36. Thrall, G., Lane, D., Carroll, D. \& Lip, G. Y. H. (2007). A systematic review of the effects of acute psychological stress and physical activity on haemorheology, coagulation, fibrinolysis and platelet reactivity: implications for the pathogenesis of acute coronary syndromes. Thrombosis Research, 120, 819-847.

37. Thombs, B. D., Bass, E. B., Ford, D. E., Stewart, K. J., Tsilidis, K. K., Patel, U., Fauerbach, J. A., Bush, D. E. \& Ziegestein, R. C. (2005). Prevalence of depression in survivors of acute myocardial infarction. review of the evidence. Journal of General Internal Medicine, 21 (1), 30-38.

38. Wall, H. K., Ballard, J., Troped, P., Njike, V. Y. \& Katz, D. L. (2010) Impact of home-based, supervised exercise on congestive heart failure. International Journal of Cardiology, 145 (2), 267-270.

39. Warburton, D. E., Nicol, C. W. \& Bredin, S. S. (2006). Health benefits of physical activity: the evidence. Canadian Medical Association Journal, 174, 801-809.

40. Ware, J. E, Jr. (2000). SF-36 health survey update. Spine, 25 (24), 3130-3139.

41. Ware, J. E. Jr. \& Sherbourne, C. D. (1992). The MOS 36-item shortform health survey (SF-36). I. Conceptual framework and item selection. Medical Care, 30 (6), 473-483.

42. Wilson, L. K., Sánchez-Rodríguez, M. A. \& Mendoza, V. M. (2009). Sedentarismo como factor de riesgo de trastornos depresivos en adultos mayores. un estudio exploratorio. Revista de la Facultad de Medicina, UNAM, 52 (6), 244-247.

43. Yesavage, J. A., Brink, T. L., Rose, T. L., Lum, O., Hanh, V., Adey, M. \& Leirer, V. O. (1983). Developmen and validadtion of a geriatric depression screening scale: a preliminary report. Journal of Psychiatric Research, 17 (1), 37-49. 